Article

\title{
Differently Shaped Au Nanoparticles: A Case Study on the Enhancement of the Photocatalytic Activity of Commercial $\mathrm{TiO}_{2}$
}

\author{
Zsolt Pap ${ }^{1,2,3, \dagger}$, Zsejke Réka Tóth ${ }^{1, \dagger}$, Virginia Danciu ${ }^{1}$, Lucian Baia ${ }^{2,4, \dagger, *}$ and \\ Gábor Kovács ${ }^{1,2,5, \uparrow, *}$
}

1 Faculty of Chemistry and Chemical Engineering, Babeş-Bolyai University, Arany János 11, RO-400028 Cluj-Napoca, Romania; E-Mails: tothzsejkereka@yahoo.com (Z.R.T.), vdanciu@chem.ubbcluj.ro (V.D.)

2 Faculty of Physics, Babeș-Bolyai University, M. Kogălniceanu 1, RO-400084 Cluj-Napoca, Romania; E-Mail: pap.zsolt@phys.ubbcluj.ro

3 Research Group of Environmental Chemistry, Institute of Chemistry, University of Szeged, H-6720, Szeged, Tisza Lajos krt. 103, Hungary

4 Institute for Interdisciplinary Research on Bio-Nano-Sciences, Babeș-Bolyai University, M. Kogălniceanu 1, RO-400084 Cluj-Napoca, Romania

5 Faculty of Science and Informatics, Department of Applied and Environmental Chemistry, University of Szeged, H-6720, Szeged, Rerrich Béla tér 1, Hungary

$\dagger$ These authors contributed equally to this work.

* Authors to whom correspondence should be addressed; E-Mails: lucian.baia@phys.ubbcluj.ro (L.B.); gkovacs@chem.ubbcluj.ro (G.K.).

Academic Editor: Klara Hernadi

Received: 16 October 2014 / Accepted: 24 December 2014 / Published: 31 December 2014

\begin{abstract}
In the present work, the influence of a gold nanoparticle's shape was investigated on the commercially available Evonik Aeroxide P25. By the variation of specific synthesis parameters, three differently shaped Au nanoparticles were synthetized and deposited on the surface of the chosen commercial titania. The nanoparticles and their composites' morphological and structural details were evaluated, applying different techniques such as Diffuse Reflectance Spectroscopy (DRS), X-ray Diffraction (XRD), and Transmission Electron Microscopy (TEM). The influence of the Au nanoparticles' shape was discussed by evaluating their photocatalytic efficiency on phenol and oxalic acid degradation and by investigating the $\mathrm{H}_{2}$ production efficacy of the selected composites. Major differences in
\end{abstract}


their photocatalytic performance depending on the shape of the deposited noble metal were evidenced.

Keywords: titanium-dioxide; gold nanoparticles; shape-control; photocatalysis; hydrogen production

\section{Introduction}

Since the early 1970 s a large number of publications appeared regarding $\mathrm{TiO}_{2}$-based photocatalysts, exploring the intersection of different pathways of wastewater treatment and alternative energy sources. One of the solution for these challenges was found to be the investigation of the semiconductor-properties as well as of those related to the semiconductor-metal-based nanocomposites. In the intersection of these tasks, what can be found are the photocatalytic degradation of organic contaminants and hydrogen-production using noble-metal-modified $\mathrm{TiO}_{2}$.

From the time when the first report of photocatalytic water-splitting of water using $\mathrm{TiO}_{2}$ electrodes under UV-light appeared [1], a whole research-field has grown around the topic of designing more efficient semiconductor-based photocatalysts. Nowadays, developing a high-quality, effective, stable and cheap semiconductor-based photocatalyst for the remediation of global water pollution has become an emerging research field. In recent years, a large number of semiconductor-photocatalysts have been reported $\left(\mathrm{TiO}_{2}[2,3], \mathrm{ZnO}[4,5], \mathrm{CdSe}[6,7], \mathrm{SnO}_{2}[8,9], \mathrm{CuO}[10,11], \mathrm{WO}_{3}[12,13]\right.$, etc.), but by far, the most studied material remained the $\mathrm{TiO}_{2}$ because of its stability, strong oxidizing power, chemical inertness, non-toxicity, low cost and environmentally nonthreatening nature [14-16]. This promising material, even if, from some point of view is close to the ideal photocatalyst, it has its own "Achilles' heels", like limited photosensitivity for the visible light and massive recombination of photogenerated charge carriers which can decrease its catalytic efficiency.

A promising approach in the improvement of the photocatalytic activity of $\mathrm{TiO}_{2}$ is the deposition of noble metal nanoparticles on the semiconductor surface, which can increase the efficiency of the charge transfer process by trapping the photogenerated electrons. Among noble metals, beside Pt and Ag, gold has gained considerable attention in the enhancement of photocatalytic activity of $\mathrm{TiO}_{2}$. Although variously shaped nanoparticles were synthetized (e.g., cubes [17], spheres [18], nanorods [19], and triangular prisms/nanoplates [20]) for numerous (e.g., electrochemical [21], plasmonic [22], antibacterial [23], biomedical [24], catalytic [25], sensor [26]) applications, part of the above mentioned types of nanoparticles have not been tested before together with titania for photocatalytic applications.

The performances of gold deposited on the surface of $\mathrm{TiO}_{2}$ depends remarkably on the selected support, strong contact with the photocatalyst, $\mathrm{Au}-\mathrm{TiO}_{2}$ ratio, particle size and shape. This is due to the fact that even if a large number of publication deals with the synthesis, characterization and photocatalytic applicability studies of different $\mathrm{TiO}_{2}$-based materials, the "legendary" $\mathrm{P} 25$, which is available for day-by-day use, cheap and it has generally remarkable photocatalytic property, looks to be hard to "beat", therefore we have chosen to omit the first aspect from the present work. The second aspect, namely the contact between the nanoparticles (NP) and the photocatalyst is one of the most important factor, being observable especially in the case of gold containing mesoporous $\mathrm{TiO}_{2}$, where the 
gold made possible a more efficient charge separation of electron-hole pairs, increasing their lifetime, creating effective traps for electrons due to the formation of a Schottky-barrier at the metal-semiconductor surface, the major requirement being the intimate contact of gold with titania [27,28]. On the other hand, it was observed that in the case of the $\mathrm{TiO}_{2}-\mathrm{Au}$ samples prepared by photodeposition, the activity decreased with the addition of gold, probably due to the shadowing and blocking of $\mathrm{TiO}_{2}$ active sites by bigger and scarcely effective gold nanoparticles [29], similar effect being observed for P25-Au composites, during photocatalytic degradation of phenol under UV irradiation [30]. It has to be mentioned that generally the activity of $\mathrm{Au}-\mathrm{TiO}_{2}$ composites increases as the particle size of gold decreases, reaching a maximum value of two-fold enhancement compared to pure $\mathrm{TiO}_{2}$, the effect observed during photodegradation of oxalic acid [31].

On the other hand, the effect of different geometries of gold nanoparticles was already summarized/studied systematically in various application, for targeted drug delivery and cancer treatment [32], therapeutical/phototermal effect [33], DNA detection [34], catalytic effect on CO oxidation [35] but to the best knowledge of the authors the effect of the various structures was not studied and correlated with their structural, photocatalytic properties and $\mathrm{H}_{2}$ productivity.

Thus, the main aim of this work was to investigate the impact of differently shaped gold nanoparticles deposited on the surface of commercial $\mathrm{TiO}_{2}$ via various methods (Diffuse Reflectance Spectroscopy (DRS), X-ray Diffraction (XRD), and Transmission Electron Microscopy (TEM)) and to correlate these with the obtained photocatalytic activity for various model organic contaminants, taking into account the intermediates' evolution (of phenolic compounds) and $\mathrm{H}_{2}$ production.

\section{Results and Discussion}

\subsection{The Proposed Research Plan}

As already detailed in the introduction, the shape control of nano-sized gold is rather important, as some of the properties are exploitable only at a specific crystallite geometry. In the present work, differently shaped gold nanoparticles were obtained taking in count the dimensionality aspects, which were:

(a) Spheres: 3D particles described by a single dimension: the radius of the sphere;

(b) Triangles: 2D particles described by multiple geometric elements and for which one of the geometry defining element is significantly smaller than the other;

(c) Wires: 1D particles where two of the geometry defining element is significantly smaller than the other.

At first sight these nanoparticles seem unrelated to each other. However, the synthesis was chosen in such way that the weight of the differently shaped gold nanoparticles were in the same range (i.e., the weight of a $15 \mathrm{~nm}$ thick and $3 \mu \mathrm{m}$ long nanowire is nearly the same as the weight of a $60 \mathrm{~nm}$ photoreduced gold nanoparticle). This was rather important as the conduction of the photogenerated electrons can occur in a different way for each type of material. To verify if this aspect is indeed true, $1 \mathrm{wt} \%$ Au containing Aeroxide P25 composites were obtained. Their photocatalytic activity was verified for the degradation of two different substrates (oxalic acid and phenol). Additionally, the photocatalytic hydrogen production capacity was also evaluated. The whole research plan is represented schematically in Figure 1. 


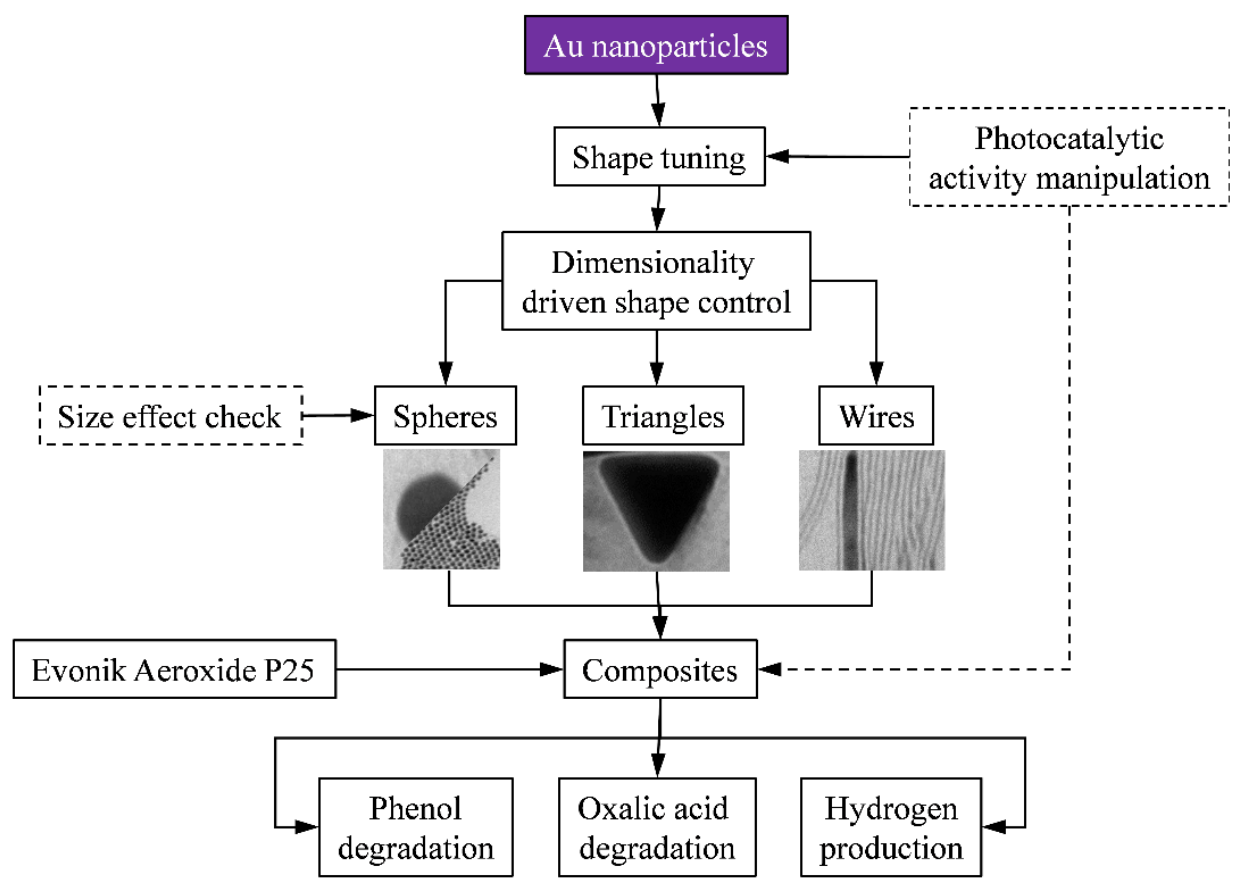

Figure 1. Schematic diagram of the applied research strategy.

\subsection{Characterization of the Photocatalysts}

\subsubsection{X-ray Diffraction (XRD)}

As a first step of characterization process, the crystal size and phase composition was evaluated using diffraction patterns. No changes were observed in these parameters of the composites comparing to P25 (Figure 2).
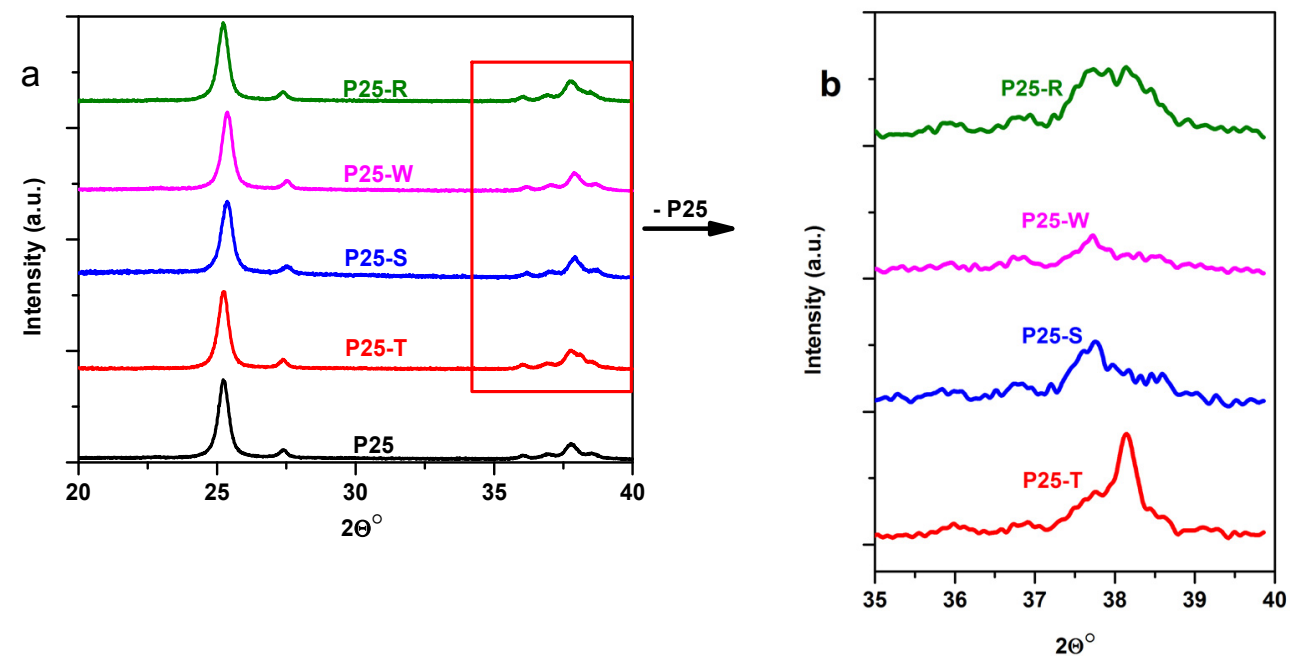

Figure 2. Diffraction patterns of bare and Au-modified P25 composite materials (a) and difference signals between P25 and the composites (b) (for the abbreviations of the samples, please consult Section 3.3-Synthesis of $\mathrm{Au}-\mathrm{TiO}_{2}$ composites).

As expected, the X-ray diffraction patterns confirmed the particle size and crystal phase composition of P25 known from literature (89 wt\% anatase, $11 \mathrm{wt} \%$ rutile; $25-40 \mathrm{~nm}$ primary particle size) [36]. 
Taking into consideration that the studied composite materials also contains gold it would be interesting to see if a shape dependence can be discovered in the diffraction patterns of Au. In order to analyze in-detail the diffraction patterns of Au NPs, difference diffractograms were generated by subtracting from the Au-composites' diffraction signal the "reference" P25 diffraction pattern (in the interval between $35^{\circ}$ and $40^{\circ}\left(2 \Theta^{\circ}\right)$ ). The chosen $2 \Theta$ interval is of interest because the most intensive peak of $\mathrm{Au}$ can be found at $38^{\circ}\left(2 \Theta^{\circ}\right)$, for the (111) crystallographic plane [37]. The relatively narrow range, in which the diffractograms were recorded (only between $20^{\circ}$ and $40^{\circ}\left(2 \Theta^{\circ}\right)$ ), is explained by the fact that other diffraction peaks for $\mathrm{Au}$ (e.g., at $43^{\circ}\left(2 \Theta^{\circ}\right)$ for facet $(200)$ or $(220)$ at $64^{\circ}\left(2 \Theta^{\circ}\right)$ ) are usually 3-4 times less intensive than the signal for Au facet (111) [37]. Even this mentioned signal was barely observable due to the low concentration (1\%) of Au NPs in the composite materials.

Taking into account these circumstances, it can be concluded that in the case of P25-T composites the (111) facet's signal is clearly visible at $38^{\circ}\left(2 \Theta^{\circ}\right)$, as this is the dominant facet in the case of triangle shaped Au nanoparticles [38]. In the case of P25-W this signal is diminished due to the less dominant presence of the mentioned crystallographic plane [39]. For the P25-S and P25-R spherical composites these signals are less visible due to the polycrystalline character of the nanoparticles. It should be noted here that the peak at $37.6^{\circ}\left(2 \Theta^{\circ}\right)$ is a glitch appearing due to the differentiation process.

\subsubsection{Transmission Electron Microscopy (TEM)}

In order to have a clearer overview about the above mentioned Au nanoparticles and the P25-based composites, TEM micrographs were acquired from the "pure" colloidal $\mathrm{Au}$ nanoparticles and P25-based composites.

The obtained micrographs have shown that the main target, namely, to obtain differently shaped Au NPs was successfully achieved as already "foretold" by the diffraction patterns. As a result of the first synthesis, relatively small and uniform Au nanospheres were obtained, with a diameter of $\approx 8-9.9 \mathrm{~nm}$ (72\%). Lowering the temperature of the synthesis, as it was already described in Section 2.2 induced the formation of Au nanowires. As it can be observed in Figure 3, these nanowires had a width between 8 and $10 \mathrm{~nm}$ and length of $\approx 1-2 \mu \mathrm{m}$. It can be also observed that this sample, containing mostly nanowires also contained a small amount of spheres $(81 \%$ vs. $19 \%$ weight distribution between two geometries). From the "contamination" with spheres, from the "fractures" observable on the nanowires and from the fact that the single difference of the synthesis pathways was the lower temperature in the case of nanowires, it can be concluded that the wires are "grown" from the nanospheres (Figure 3).

In order to be able to correlate the size-dependence of the Au NPs and the photocatalytic activity in the further investigations, larger spherical nanoparticles were also synthetized using the "traditional" photodeposition method, in presence of P25 (therefore, just micrograph of its composite can be shown) [40]. Using this method, we successfully reproduced the spherical Au-nanoparticles, already described, with a diameter of $50 \mathrm{~nm}$. For the third sample it was observable that the synthesis of nano-triangles was successful (equilateral triangles were obtained with size measuring between 60 and $100 \mathrm{~nm}, 78 \%$ ). Besides triangles smaller amounts of cubes and rhombs $(22 \%)$ were detected, probably from the simultaneous twin-growth of two triangles, pentagonal (10\%) and polyhedral (12\%) particles. 


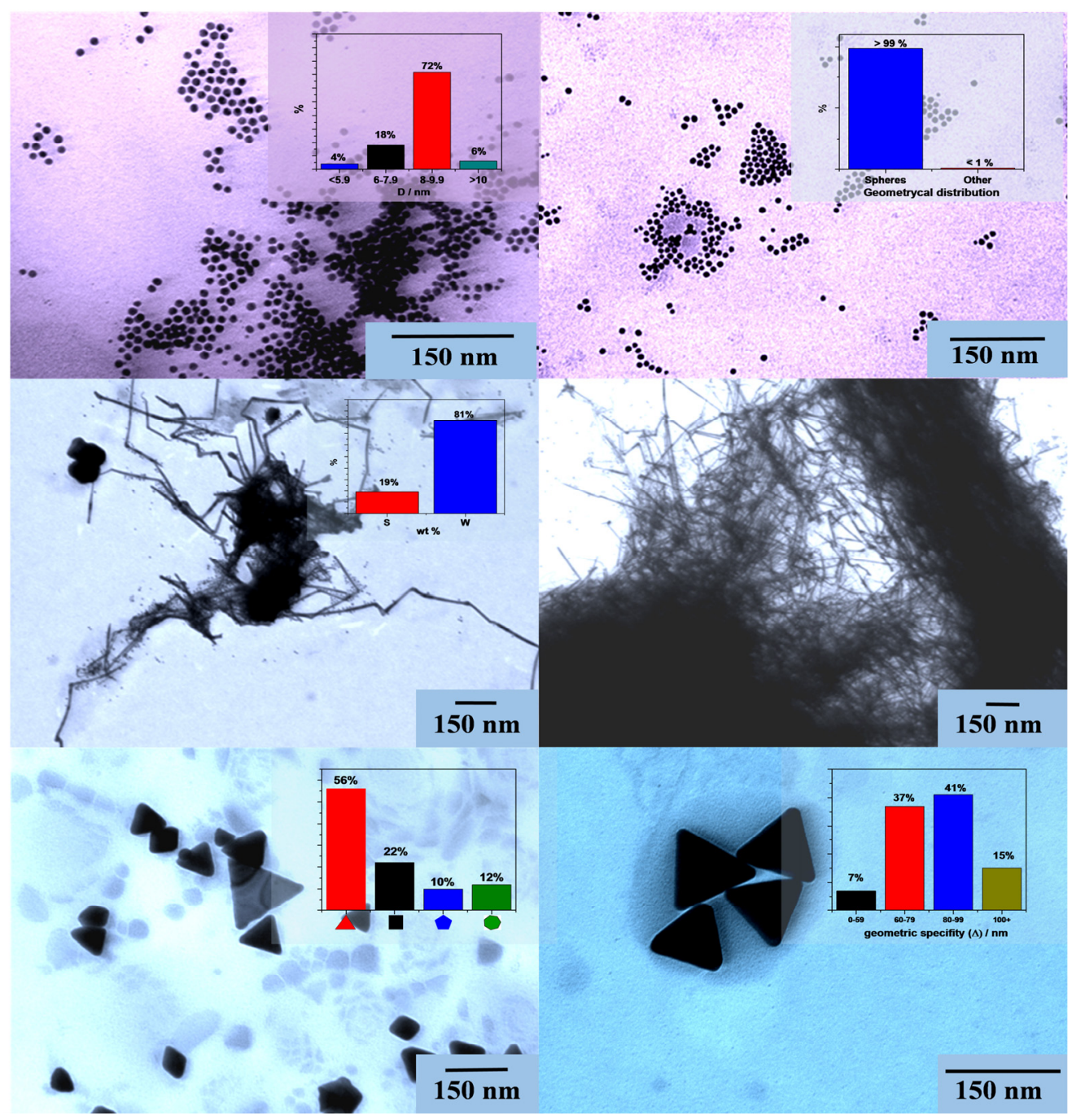

Figure 3. TEM micrographs of differently shaped Au-NPs (rows from up to down: spheres, wires, triangles).

TEM micrographs were taken also from $\mathrm{TiO}_{2}-\mathrm{Au}$-based nanocomposites. On this micrographs the Au NPs can be once more observed, this time in their composite form, alongside the titania photocatalyst. Analyzing in-detail these connections between $\mathrm{Au}$ and $\mathrm{TiO}_{2}$ nanostructures and the morpho-structural particularities of the Au NPs, working mechanisms were proposed in order to make predictions for the photocatalytic activities of the composites. In the first case, when smaller spherical NPs (case of P25-S) were introduced in the composites, the generated electron-hole pairs can be separated more efficiently if the particle size is sufficiently low. This is possible due to the higher ratio of Au nanoparticles/TiO 2 nanocrystallites which favors the efficient charge separation mechanism. Thus, as more NPs are loaded on the surface of the photocatalyst, this behavior being showed in the literature as well in previous studies [41,42]: In the case of P25-R composite the photocatalytic activity is supposed to be lower because the size of the spherical particles are higher, around $60 \mathrm{~nm}$.

For the P25-W composite the situation differs completely, observing the connection between multiple P25 particles and the lengthiness of the Au NPs (around $1 \mu \mathrm{M}$ ) that the multiple electrons are "taken" away by the nanowires from the $\mathrm{e}^{-} / \mathrm{h}^{+}$-pairs, but also a higher recombination rate is hypothetically possible due to the high possibility to "meet" a new $\mathrm{TiO}_{2}$ particle causing "nano-short-circuiting". 
Similarly, a lower photocatalytic rate can occur when larger triangles build up the composites (P25-T), where more P25 particles are attached to the same Au particle. This means that the photogenerated electrons are separated efficiently in the first instance, however, there is an increased probability for the recombination process due to the high $\mathrm{TiO}_{2} / \mathrm{Au}$ triangle ratio (Figure 4). The theories describing the mechanisms of charge-carriers listed above were "tested" on the photocatalytic measurements using phenol and oxalic acid as model-contaminants.
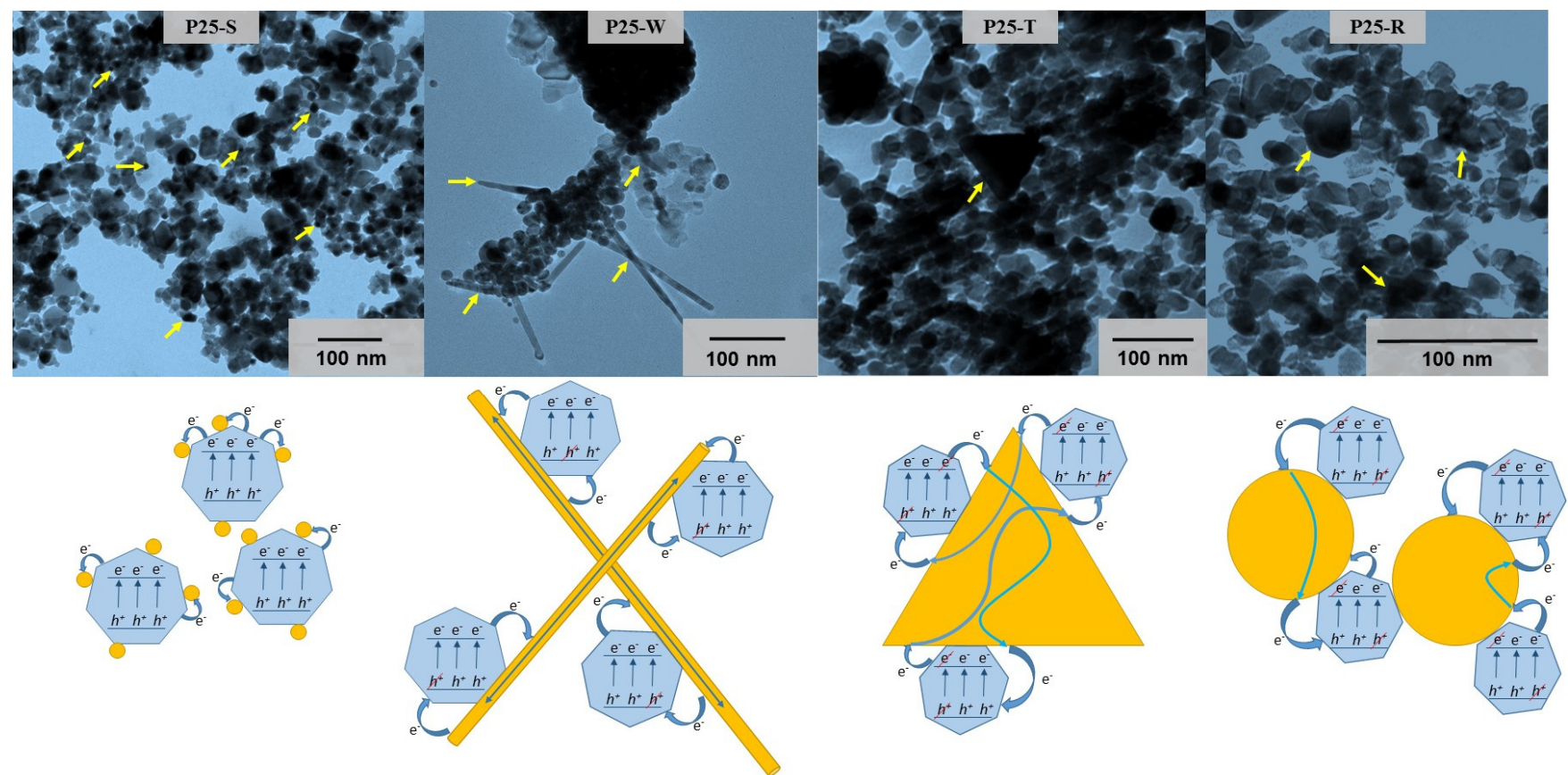

Figure 4. TEM micrographs and schematic representation of $\mathrm{P} 25-\mathrm{Au}$ composites (P25-S, P25-W, P25-T and P25-R).

\subsubsection{Diffuse Reflectance Spectroscopy (DRS)}

The next critical parameter, from the point of view of the photocatalytic activity, was investigated, namely, the light-absorption properties of the Au NP-based composites.

The obtained $\mathrm{Au}$ NPs size and shape was also observable in reflectance spectra due to the surface plasmon resonances visible between 520 and $580 \mathrm{~nm}$ [43], showing a substantial, shape and size dependent difference in this region. It can be observed that the less intense plasmonic bands, also shifted slightly to lower wavelengths can be observed in the case of P25-S and P25-R, while samples containing larger $\mathrm{Au}$ nanoparticles (P25-W and P25-T) have their characteristic plasmonic band at higher wavelengths. This can be reasonably attributed to the fact that for the composites with larger nanoparticles the absorption band broadens extending to the all visible range, whereas for smaller $\mathrm{Au}$ NPs, the band intensity decreases, becoming close to "flat" in the case of composite with spherical gold.

As it can be seen in Figure 5, the deposition of differently shaped Au particles induced significant changes in the optical properties of P25; consequently, the evaluation of the band-gap values was mandatory. Using the Kubelka-Munk equation, it was found that lowering the band-gap value by modifying the pure P25 (3.11 eV) with noble-metal was successful, the lowest value being associated with P25-S (2.70 eV), with a slightly higher value for P25-R $(2.80 \mathrm{eV})$, while P25-W and P25-T had a 
band-gap value of $2.85 \mathrm{eV}$. This means that the alteration of the Au-shape can help for a fine tuning of the optical properties, enables a careful design of a photocatalyst with a desired band-gap.

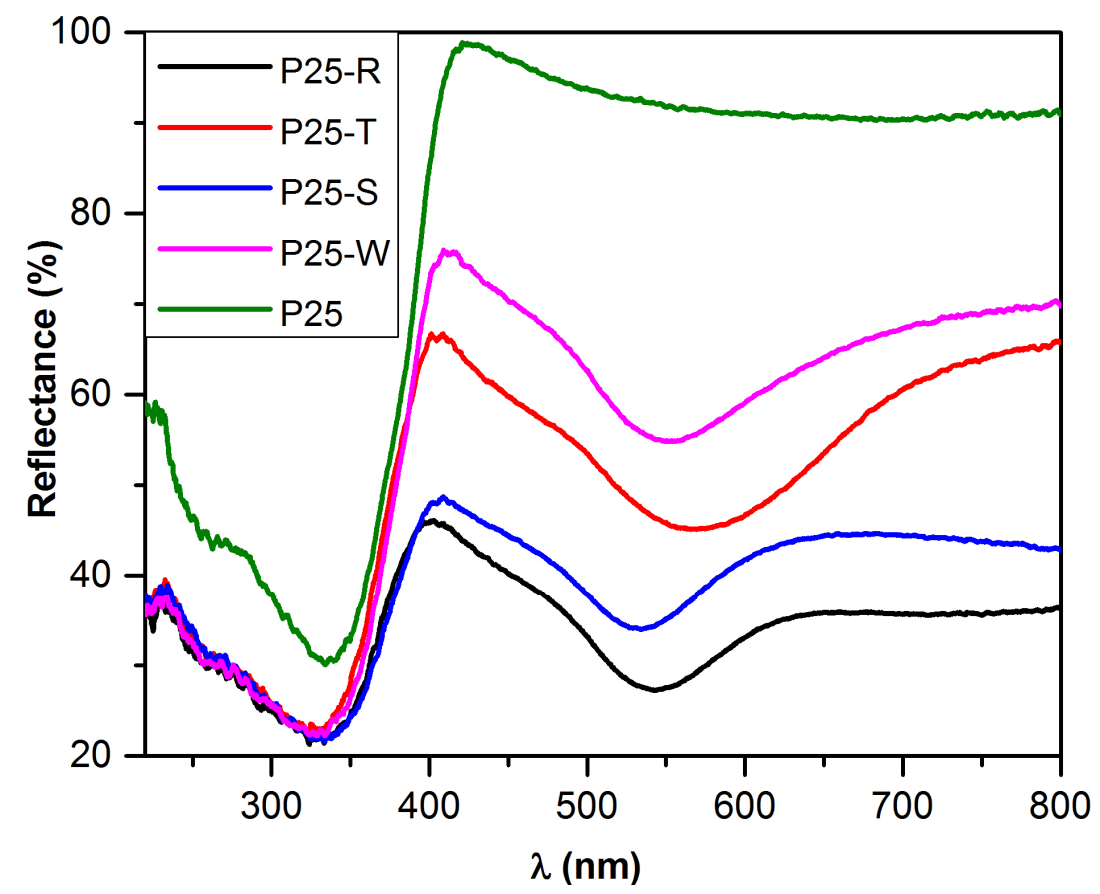

Figure 5. Comparison of different DRS spectra containing differently shaped Au NPs.

Analyzing the first order derivative of the reflectance's of the composites and the pure P25 itself, two electronic transitions can be observed, both being responsible for the UV light absorption of the catalysts, at $\approx 365$ and $\approx 400 \mathrm{~nm}[44,45]$. These peaks in terms of energy, are corresponding to band-gap values of anatase and rutile. In addition to the qualitative presence of these transitions, it is clearly visible that the addition of differently shaped Au NPs to the commercial titania induces a change in the ratio of these peaks. It can be observed in the first order derivative spectra of the composites that the ratio of anatase/rutile transitions is inverted (1/1.25 for P25 shifts to ratios around 1/0.33 and 1/0.75 in the case of composites). This behavior could be explained with the fact that the deposited gold NPs are taking the photogenerated electrons from the anatase, giving less importance to the presence of the rutile phase in the composite. The lowest $\mathrm{A} / \mathrm{R}$ electron transition ratio is present in the sample $\mathrm{P} 25-\mathrm{S}$, this can be attributed to the smaller Au particle's size which can take even more electrons from the charge separation process. It can be also observed that not only the ratio, but also the position of the electron transition band, is affected by the deposited Au nanoparticles. While the position of the peak is quite similar in the case of P25 and P25-S (365 and $395 \mathrm{~nm}$ ), the addition of large triangular shaped nanoparticles shifts both transition to higher energy value/lower wavelengths (360 and $390 \mathrm{~nm}$ ). The P25-W composite exhibits a change in the "other direction", the peaks can be found at higher wavelengths, at 370 and $400 \mathrm{~nm}$ (Figure 6). 

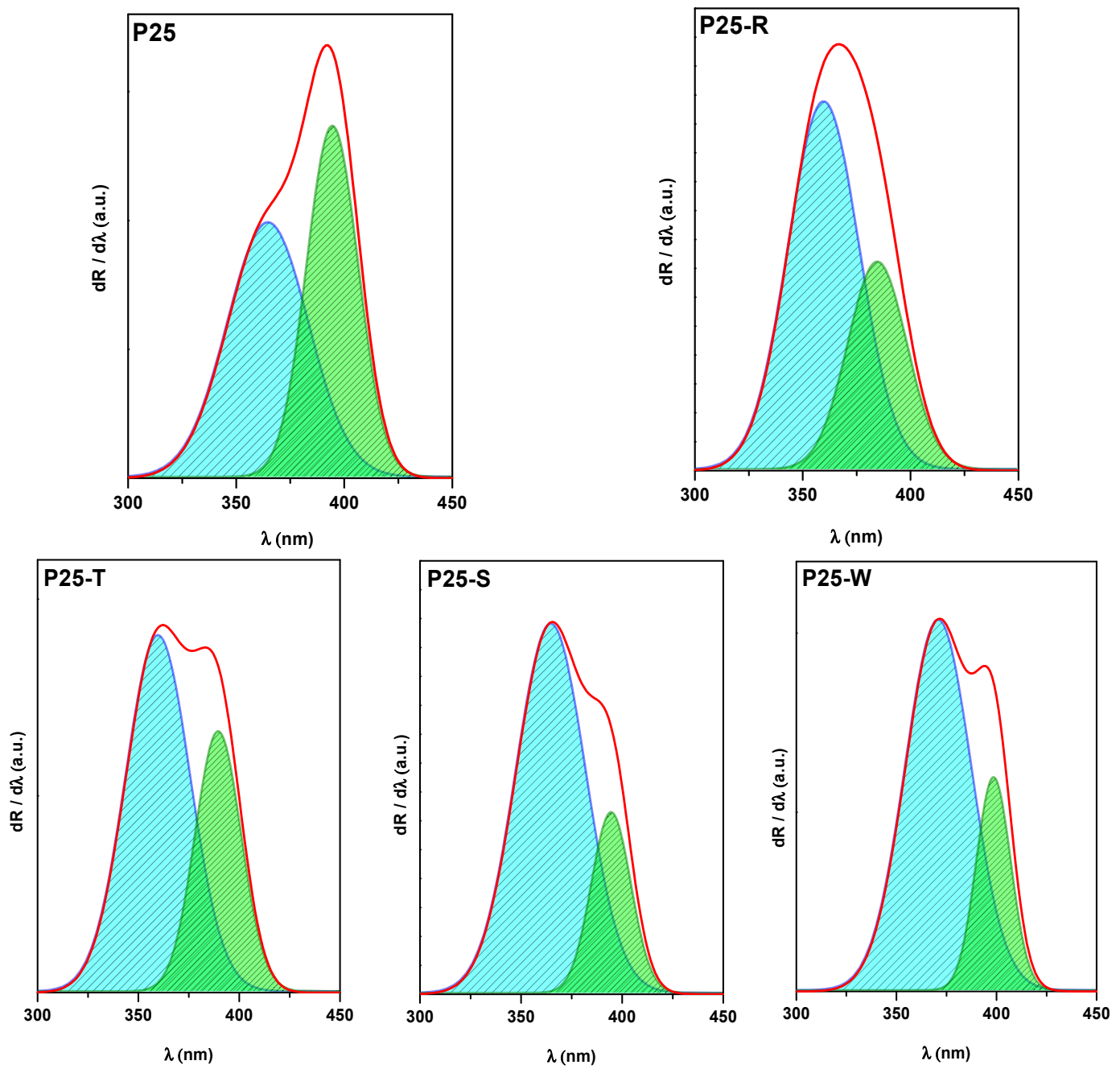

Figure 6. First order derivative spectra of P25-based nanocomposites.

\subsection{Photocatalytic Performance of the Obtained Nanocomposites}

In the previous section, we have shown that the shape of a noble metal nanoparticle influences greatly the composite's overall band-gap. Thus, a series of photocatalytic measurements were performed in order to evaluate the activity differences, which could be caused by the shape and dimension of the Au-nanoparticles.

\subsubsection{Photodegradation of Phenol}

The photodegradation of phenol was carried out under UV irradiation ( $\lambda \max \approx 365 \mathrm{~nm}$ ). UV irradiation was chosen in order to distinguish the effect of the charge separation efficiency of the gold NPs, phenomenon which is more efficient using UV irradiation, carefully eliminating the effect of any plasmon-resonance-based optical interaction (e.g., excitation, etc.). Our experiments regarding gold deposited titanias showed that the presence of the gold nanoparticles are inhibiting the phenol decomposition rates, fact was already was observed in our previous works [31], but they are maintaining a comparable efficiency in terms of degradation rate $(50.5 \%-71 \%$ in $2 \mathrm{~h}$, comparing to the $82.4 \%$ of $\mathrm{P} 25)$. It is already known that for the photocatalytic decomposition of phenol the $\mathrm{OH} \cdot$ radicals are responsible. Therefore, their generation rate is a crucial aspect, which is mainly determined by the charge 
separation efficiency and the nature of the substrate (adsorbing/non-adsorbing). As it was already shown in Figure 4, the gold NPs in some cases can inhibit or enhance the charge carrier separation process, depending on:

(i) The particle's size in the base catalyst - in the present study this was ruled out by using Aeroxide P25 in all the composite materials;

(ii) Au NPs crystal size — all the Au NPs obtained are large Au NPs (except for P25-S).

In our case the larger gold NPs enhances the charge recombination rate (as discussed in Section 2.2.2), by conducting the electrons from a $\mathrm{TiO}_{2}$ particle to another one. Hence, the possibility to obtain photo-generated charge carriers was lowered, thus inhibiting $\mathrm{OH} \cdot$ generation radical as well (this is why all the Au containing catalyst have lower activity towards phenol). However, not all the Au geometries favor the recombination in the same way, as was already proposed in Figure 4.

It would seem that the most "detrimental" recombination mechanism is the one described for the sample P25-T (50.5\% phenol degradation yield). The "triangle" shaped Au nanoparticles are interconnecting several $\mathrm{TiO}_{2}$ crystals, resulting an interparticular charge neutralization, inhibiting significantly the possibility to generate reactive OH radicals. Surprisingly, small sized spherical Au NPs were also not effective in the degradation of phenol (P25-S, 52.9\% degraded phenol). The latter one could mean that the degradation intermediates are seriously intervening in the degradation mechanism as already demonstrated in our recent work [44,45], although the charge separation mechanism is considered to be efficient at this Au particle size [31]. A significant inhibition of the phenol removal was also registered in the case of $\mathrm{P} 25-\mathrm{R}$ (58.4\% of removed phenol), where the charge separation scenario was considered similar to the one proposed for P25-T. The main difference was in the electron transfer rate, which is dependent on the crystal geometry - a critical issue that is currently under investigation. The least inhibitive effect on the phenol-degradation was observed in the case of P25-W (71\% of degraded phenol). Thus, the Au nanowire's (NW) charge separation is the most effective, which should be reflected also in the oxalic acid degradation.

In terms of the reaction rate, the situation changes. The best performing composite was the P25-W, reaching a $7.45 \times 10^{-3} \mathrm{mM} \cdot \mathrm{min}^{-1}$ (value calculated from the first five points of the degradation process), which was higher than the pure industrial titania. The difference in the terms of efficiency measured in the degradation yield and reaction rates resides in the fact that Au NPs are playing a crucial role in the accumulation of hydroxylated intermediates. These compounds are usually detrimental for the photocatalytic efficiency $[44,45]$. Consequently, in the beginning of the degradation process the phenol is degraded efficiently on P25-W (even more efficient then the bare P25). As the degradation intermediates are accumulating, the phenol degradation is inhibited and the degradation kinetics are slowly changing (the shape of the degradation curve P25-W, which intersects the curve of P25-Figure 7). As it was already shown in Figure 4, the gold NPs in some cases can inhibit or enhance the charge carrier separation process, depending on the particle's size of the base catalyst and Au NPs as well. In our case, the larger gold NPs enhances the charge recombination rate, by conducting the electrons from a $\mathrm{TiO}_{2}$ particle to another one. Hence, the possibility to obtain photo-generated charge carriers was lowered, thus inhibiting $\mathrm{OH} \cdot$ generation radical as well (this is why all the $\mathrm{Au}$ containing catalyst have lower activity towards phenol). The other composites showed a somewhat lower photocatalytic performances: P25-T and P25-R $\left(5.71 \times 10^{-3} \mathrm{mM} \cdot \mathrm{min}^{-1}, 5.01 \times 10^{-3} \mathrm{mM} \cdot \mathrm{min}^{-1}\right.$ and $4.49 \times 10^{-3} \mathrm{mM} \cdot \mathrm{min}^{-1}$, 
respectively). The less efficient catalyst was the $\mathrm{P} 25-\mathrm{S}$, its reaction rate was lower by $40 \%$ than the best performing P25-W (Figure 7 and Table 1).

It has to be mentioned, that the photolysis under UV irradiation was $\approx 5.6 \%$, value smaller with approximately one order of magnitude, compared to the results obtained using the P25-Au composites.

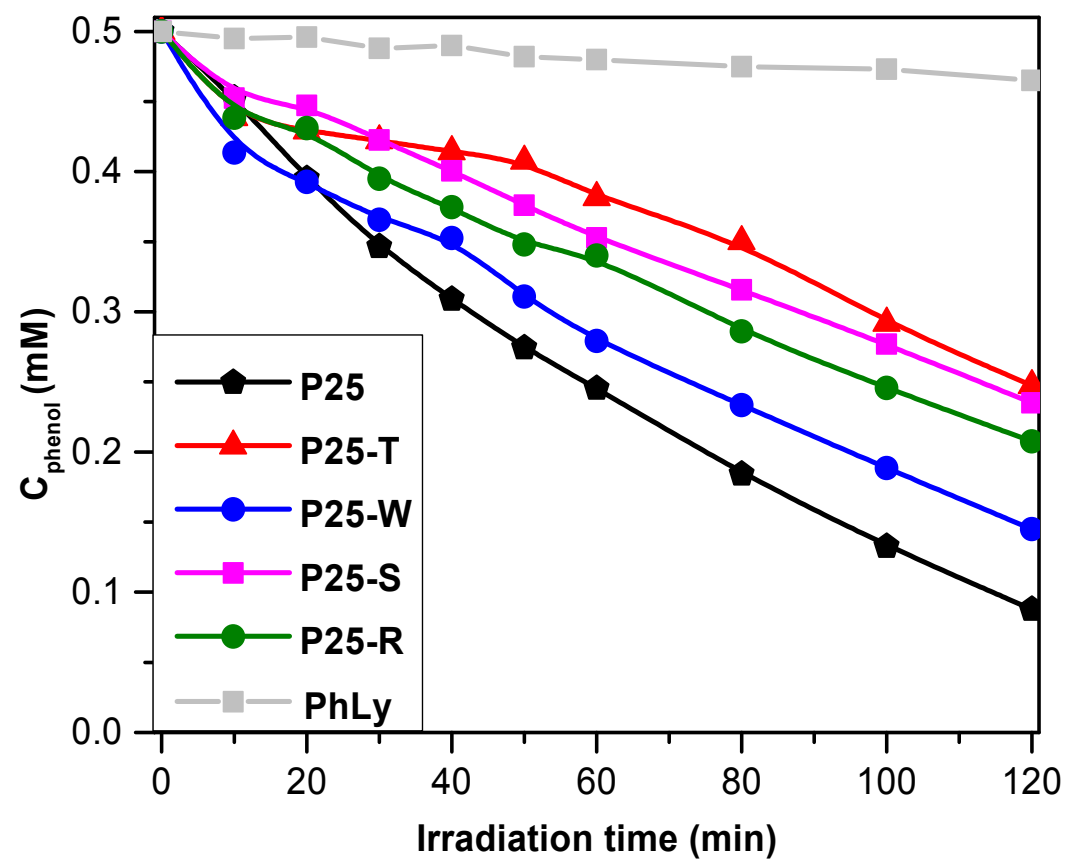

Figure 7. Phenol degradation curves of the prepared nanocomposites under UV irradiation (PhLy refers to the degradation of phenol without photocatalyst).

Table 1. Photocatalytic efficiencies of P25-based composites in different photocatalytic applications.

\begin{tabular}{ccccccc}
\hline Sample & $\begin{array}{c}\text { ro,oxalic acid } \\
\left(\mathbf{m M} \cdot \mathbf{m i n}^{-\mathbf{1}}\right) \\
\times\left(\mathbf{1 0}^{-\mathbf{3}}\right)\end{array}$ & $\begin{array}{c}\text { ro, phenol } \\
\left(\mathbf{m} \mathbf{M} \cdot \mathbf{m i n}^{-\mathbf{1}}\right) \\
\times\left(\mathbf{1 0}^{-\mathbf{3}}\right)\end{array}$ & $\begin{array}{c}\text { Oxalic aciduv/Vis } \\
\text { degradation } \\
\text { rate } \mathbf{( \% )}\end{array}$ & $\begin{array}{c}\text { Phenoluv/Vis } \\
\text { degradation } \\
\text { rate } \mathbf{( \% )}\end{array}$ & $\begin{array}{c}\mathbf{H}_{2} \\
\text { production } \\
\text { rate }\left(\mathbf{m L} \cdot \mathbf{h}^{-\mathbf{1}}\right)\end{array}$ & $\begin{array}{c}\text { Band gap } \\
(\mathbf{e V})\end{array}$ \\
\hline P25-W & 46.0 & 7.45 & $97.4 / 21.0$ & $71.0 / 12.2$ & 0.62 & 2.85 \\
P25-S & 71.7 & 3.19 & $97.6 / 20.8$ & $52.9 / 8.6$ & 0.78 & 2.70 \\
P25-R & 51.2 & 4.49 & $86.2 / 19.1$ & $58.4 / 9.5$ & 0.63 & 2.80 \\
P25-T & 69.0 & 5.01 & $75.5 / 16.8$ & $50.5 / 7$ & - & 2.85 \\
P25 & 41.9 & 5.71 & $54.3 / 12$ & $82.4 / 13.8$ & - & 3.11 \\
\hline
\end{tabular}

As it was predictable, the less efficient composite for photodegradation was P25-T due to the larger $\mathrm{Au}$ NPs size and to the fact that more titania particles can be attached on the same noble metal particle, making the charge-separation less efficient. Similar process can explain the lower efficiency in the case of P25-W (described in detail at last part of Section 2.2.2).

It has to be mentioned, that photocatalytic tests were made in order to determine the efficiencies of the composites under visible light irradiation (Table 1). It can be concluded that the tendencies are similar regarding the degradation rates, but the efficiencies are generally more than 5 times smaller than those observed in UV. Therefore, due to the low degradation rates and the possible errors of measurements, the results were no longer discussed (see Supplementary Materials). 


\subsubsection{Photodegradation of Oxalic Acid and Photocatalytic Hydrogen Production}

In order to have information about the photodegradative properties of $\mathrm{P} 25$-Au composites in removal of non-aromatic, simple organic compounds, photodegradation measurements were carried out on oxalic acid as model contaminant, which is a well-known, good absorbing organic substrate.

As it can be seen on Figure 8, all the composites have shown an increased efficiency compared to P25 due to the fact that the oxalic acid molecule is adsorbing on the surface of the catalyst, trapping in this way more efficiently the photogenerated holes (that is also the reason why oxalic acid is used as a hole-scavenger molecule in photocatalytic measurements and $\mathrm{H}_{2}$-production). Speaking in term of reaction rates, the best performing catalyst was the $\mathrm{P} 25-\mathrm{S}$, achieving a reaction rate of $71.7 \times 10^{-3} \mathrm{mM} / \mathrm{min}$, followed by the P25-T, P25-R. The least efficient composite was the one made with Au nanowire nanoparticles, having a $\approx 40 \%$ lower efficiency lower with but still higher than the value reached by pure P 25 .

In term of conversion after the $2 \mathrm{~h}$ experiment, the "ranking" slightly changes. The P25-S is showing the highest conversion rate but the nanowire containing composite is also converting similar amount of oxalic acid in the range of experimental error. The composite made with photodeposition, the conversion rate is still at a high rate, for $\mathrm{P} 25-\mathrm{T}$ this value is decreasing to $75.5 \%$, which is still higher with $\approx 30 \%$ than the value obtained using pure P25.

Photocatalytic tests were made also under visible irradiation, in order to determine the efficiencies of the composites (Table 1). Briefly, it can be concluded that the tendencies are similar regarding the degradation rates, but the efficiencies are generally around four times smaller than those observed in UV, if we are taken under the loupe the degradation rates. Therefore, due to the low degradation rates and the possible errors of measurements, the results were no longer discussed (see Supplementary Materials).

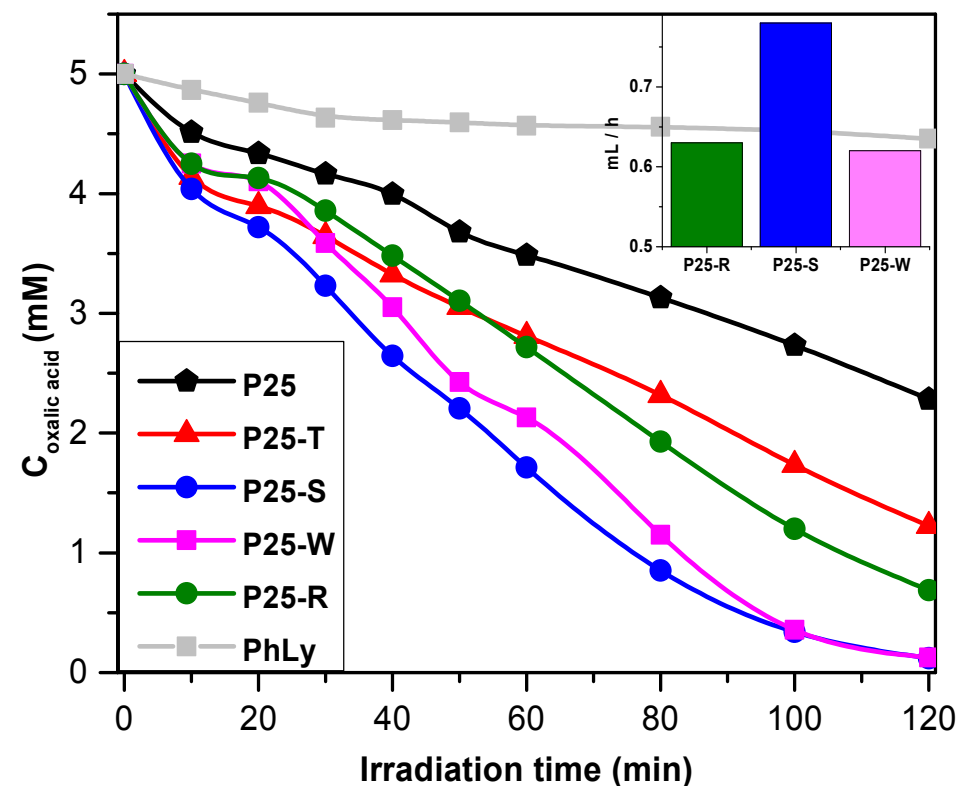

Figure 8. Photodegradation curves of oxalic acid in the presence of the prepared nanocomposites under UV irradiation and (inset) hydrogen production rates of the selected composites. 
As it was already shown in the previous sections, the shape of the Au nanoparticles is vital in the definition of the photocatalytic activity of a given composite material. Hydrogen production measurements were carried out, in order to find out if the shape of the Au nanoparticles' is indeed a determining factor in this case.

In order to determine the hydrogen production rate, oxalic acid $(50 \mathrm{mM})$ was used for photocatalytic decomposition in oxygen-free conditions. The $\mathrm{H}_{2}$ production rate was calculated using data collected from the interval between 30 and $90 \mathrm{~min}$, a time when the productivity of composites has reached a quasi-stationery rate. As it is shown in Table 1 and in Figure 8 (inset), the highest hydrogen production rate was achieved by the composite containing small spherical Au nanoparticles, fact expected looking at the results obtained by photodegradation of oxalic acid. The wire-shaped Au NPs and the NPs obtained by photodeposition had shown a lower activity, producing $\approx 0.62 \mathrm{~mL} \cdot \mathrm{h}^{-1}$, presenting a lower difference between their efficiencies as expected from efficacy toward oxalic acid (see also Supplementary Materials). P25-T was omitted due to its relatively low activity toward oxalic acid, shown in previous section and pure P25 was already shown inactive in previous studies in $\mathrm{H}_{2}$ production studies.

\section{Experimental Section}

\subsection{Materials}

All chemicals used were of analytical grade. Trisodium-citrate was purchased from Alfa-Aesar (Karlsruhe, Germany) while the gold precursor $\left(\mathrm{HAuCl}_{4} \cdot 3 \mathrm{H}_{2} \mathrm{O}\right)$, oleylamine and cetyltrimethylammonium bromide (CTAB) were purchased from Sigma-Aldrich (Steinheim, Germany). Evonik Aeroxide P25 (Essen, Germany) was used, as received, without further purification for the preparation of the composite materials.

\subsection{The Synthesis of the Au-NPs}

According to Bernardi et al. [39] gold nanowires were synthetized dissolving $100 \mathrm{mg}$ of $\mathrm{HAuCl}_{4}$ in $1 \mathrm{~mL}$ of ethanol. The ethanolic solution of the precursor was added to $25 \mathrm{~mL}$ of oleylamine preheated at $75^{\circ} \mathrm{C}$. The mixture's temperature was kept for $6 \mathrm{~h}$ in a nitrogen atmosphere (without stirring). During the synthesis the color of the solution was changed from transparent to orange trough purple. Finally, a red precipitate was obtained. After $6 \mathrm{~h}$ the reaction product was centrifuged and washed with ethanol (with $3 \times 25 \mathrm{~mL}, 1 \times 16 \mathrm{~min}$ and $2 \times 8 \mathrm{~min}$, all at $4400 \mathrm{rpm}$ ) and with methanol ( $8 \mathrm{~min}, 4400 \mathrm{rpm}$ ). The nanospheres were obtained in a similar way, the only difference was that the oleylamine was heated and kept at $85^{\circ} \mathrm{C}$.

The gold nanotriangles were synthetized according to Kumar et al. [38] using two solutions: the first contained $896 \mu \mathrm{L} \mathrm{Na}_{3} \mathrm{C}_{6} \mathrm{H}_{5} \mathrm{O}_{7}(0.1 \mathrm{M})$ and $52.5 \mathrm{~mL} \mathrm{H} \mathrm{H}_{2} \mathrm{O}$ while the second solution contained $31.57 \mathrm{~mL}$ $\mathrm{H}_{2} \mathrm{O}$ with $112 \mathrm{mg} \mathrm{CTAB}$ and $3.43 \mathrm{~mL} \mathrm{HAuCl}_{4}(12.7 \mathrm{mM})$. The two solutions were added instantaneously into a pre-heated vessel (at $90{ }^{\circ} \mathrm{C}$, without stirring) [38].

\subsection{Synthesis of $\mathrm{Au}-\mathrm{TiO}_{2}$ Composites}

As it was already shown in representative publications [46], the highest photocatalytic activity of $\mathrm{Au}-\mathrm{TiO}_{2}$-based composites can be obtained at $\approx 0.5-1 \mathrm{wt} \%$ gold concentration, and due to the fact that 
the $1 \mathrm{wt} \%$ of Au nanoparticles can be efficiently detected by means of the optical spectroscopy and other methods [45,48], therefore all the P25-based composites synthetized below contained 1 wt $\%$ concentration of $\mathrm{Au}$ NPs. Applying the above described $\mathrm{Au}$ synthesis pathways the $\mathrm{TiO}_{2}-\mathrm{Au}$ composites, based on commercial titania, were prepared as follows:

P25-W was made adding $6.474 \mathrm{~mL}$ of Au nanowires suspension (freshly synthetized and dispersed in $100 \mathrm{~mL}$ of hexane) to a P25 suspension (made from $800 \mathrm{mg}$ P25 and $50 \mathrm{~mL}$ of hexane). In the final step, the suspension was dried for $24 \mathrm{~h}$ at $40{ }^{\circ} \mathrm{C}$;

P25-S was prepared using $4 \mathrm{~mL}$ of spherical Au-sol added to a suspended P25 (800 mg in $150 \mathrm{~mL})$ under vigorous stirring. The obtained mixture was dried for $24 \mathrm{~h}$, washed with acetone $(3 \times 50 \mathrm{~mL})$ and dried once more for $24 \mathrm{~h}$;

P25-T was prepared similar to P25-S, adding $83.63 \mathrm{~mL}$ of $\mathrm{Au}(\mathrm{t})$ sol in $800 \mathrm{mg} / 150 \mathrm{~mL}\left(\mathrm{H}_{2} \mathrm{O}\right) \mathrm{P} 25$ suspension, washed with water, dried for $24 \mathrm{~h}$, washed three times (with $\mathrm{H}_{2} \mathrm{O}$ ) and dried once more for $24 \mathrm{~h}$;

P25-R using the method of photoreduction, namely mixing Au-precursor $(3.22 \mathrm{~mL}, 12.7 \mathrm{mM})$ in $200 \mathrm{~mL}, 50 \mathrm{mM}$ oxalic acid solution with P25 (4 g/L). The mixture was kept under vigorous stirring, irradiated with UV light $\left(3 \times 60 \mathrm{~W}, \lambda_{\max } \approx 365\right)$ for $5 \mathrm{~h}$ and washed similarly to P25-T.

To ensure about the fact that all amount of Au NPs was impregnated successfully on the surface of the $\mathrm{TiO}_{2}$, obtaining in this way the optimal $1 \mathrm{wt} \%$ concentration of gold in the composites, after the centrifugation spectrophotometric measurements were made using the supernatant. On the adsorption spectra's no specific plasmonic resonances were observed, fact which was also observable with "naked eye", the supernatant being clear/colorless.

\subsection{Characterization Methods and Instrumentation}

X-ray diffraction (XRD) measurements were performed on a Rigaku diffractometer (Prague, Czech Republic), $\lambda_{\mathrm{CuKa}}=0.15406 \mathrm{~nm}, 40 \mathrm{kV}, 30 \mathrm{~mA}$, in the $20^{\circ}-40^{\circ}(2 \Theta)$ regime). The average diameter of the particles were estimated using the Scherrer equation. The weight fraction of anatase and rutile was calculated for each sample from the peak areas of the anatase and rutile peaks at $25.3^{\circ}(2 \Theta)$ and $27.5^{\circ}$ $(2 \Theta)$, respectively, using the relationship developed by Zhang and Banfield [47], and the crystallites average size was calculated using the Scherrer equation [48].

Transmission electron microscopic (TEM) measurements were executed to characterize the particle size and to identify the morphology of the particles. The TEM micrographs were recorded on a Philips CM 10 instrument (Amsterdam, Netherlands) operating at $100 \mathrm{kV}$ using Formvar coated copper grids.

JASCO-V650 spectrophotometer (Tokio, Japan) with an integration sphere (ILV-724) was used for measuring the DRS spectra of the samples $(\lambda=250-800 \mathrm{~nm})$. To obtain the band-gap energy the reflectance data were converted to $\mathrm{F}(R)$ values according to the Kubelka-Munk theory. The band gap was obtained from the plot of $[\mathrm{F}(R) \cdot E]^{1 / 2}$ versus energy of the exciting light $(E)$.

The hydrogen production experiments were executed in a Pyrex glass photoreactor (Szeged, Hungary) with $170 \mathrm{~mL}$ reactor volume, filled with $150 \mathrm{~mL}$ suspension) thermostated at $25{ }^{\circ} \mathrm{C}$ and surrounded by $10 \times 15 \mathrm{~W}$ fluorescent, low pressure mercury lamps $\left(\lambda_{\max } \approx 365 \mathrm{~nm}\right)$. The suspension's concentration was $1.0 \mathrm{~g} / \mathrm{L}$ and the applied sacrificial agent was oxalic acid $(50 \mathrm{mM})$. During the photocatalytic runs the suspension was continuously purged with $\mathrm{N}_{2}(50 \mathrm{~mL} / \mathrm{min})$ to avoid the presence of $\mathrm{O}_{2}$. The $\mathrm{H}_{2}$ gas evolved was determined with a Hewlett-Packard 5890 type gas chromatograph (New 
York, NY, USA) equipped with a thermal conductivity detector, using a $2 \mathrm{~mL}$ sampling loop (sampling times: in the first hour $10 \mathrm{~min}$, in the second hour $20 \mathrm{~min}$ ). On the basis of the $\mathrm{H}_{2}$ concentrations determined by gascromatography from the flow rate of the $\mathrm{N}_{2}$, the rate of $\mathrm{H}_{2}$-evolution $(r)$ at the time of the sampling has been determined. The total amount of produced hydrogen was estimated by integrating the area under the hydrogen evolution curve. The duration of the experiment was $2 \mathrm{~h}$.

The performance of the catalysts prepared was characterized by using the photocatalytic decomposition of phenol in solution using an open tube, double walled photoreactor $(100 \mathrm{~mL})$, surrounded by a thermostating jacket $\left(25^{\circ} \mathrm{C}\right)$. The continuously stirred reactor was surrounded and irradiated by six fluorescent lamps). The continuously stirred reactor was surrounded and irradiated by six fluorescent lamps ( $6 \mathrm{~W}$ power, $\lambda_{\max } \approx 365 \mathrm{~nm}$ ). The Vis photoreactor $\left(400 \mathrm{~mL}, c_{\mathrm{P} 25-\mathrm{Au}}=1.0 \mathrm{~g} \cdot \mathrm{L}^{-1}\right)$ is an immersion type HERAEUS Reactor system equipped with an OSRAM metal halide lamp (Power Star HCl-TC 75W/WDL type, Istanbul, Turkey) with a $\mathrm{NaNO}_{2}$ solution $(1 \mathrm{M})$ in a thermostating jacket absorbing UV photons $(\lambda<400 \mathrm{~nm})$ [30]. The concentration of phenol and oxalic acid was measured with an High Performance Liquid Chromatography consisting of a Merck-Hitachi L-7100 low-pressure gradient pump (Tokio, Japan) equipped with a Merck-Hitachi L-4250 UV-vis detector and a Lichrospher RP 18 column applying methanol/water mixture and $\mathrm{H}_{2} \mathrm{SO}_{4}$, respectively as eluent. Detection wavelength was set at the lower wavelength absorption maximum of phenol (i.e., $\lambda=210 \mathrm{~nm}$ ), the high molar absorption coefficient of which makes the detection of small quantities of the substrate possible.

\section{Conclusions}

The present work shows a systematic overview concerning the importance of the shape of Au nanoparticles in photocatalytic applications. It was shown that by adjusting the reaction parameters during Au NPs production, obtaining differently shaped particles and introducing them in composites can change considerably the optical properties of the materials, including the electron transition bands.

Additionally, the activity was also dependent on the shape of the Au nanoparticles. Generally; it can be concluded that in the case of phenol, the addition of Au NPs can decrease the efficiency of commercial catalyst, while for a good adsorbing substrate, the efficiency can be increased by $\approx 100 \%$ (degradation rate obtained at the end of $2 \mathrm{~h}$ measurement). For hydrogen production, the shape-dependence was also proved, e.g., the differences occurring for oxalic acid degradation in the case of composites containing Au nanowires and nanotriangles were diminished.

It can largely be concluded that the shape of the deposited nanoparticles can highly influence the efficiency of the composites, making possible a shape defined application tuning.

\section{Acknowledgments}

This research was supported by the European Union and the State of Hungary, co-financed by the European Social Fund in the framework of TÁMOP-4.2.4.A/ 2-11/1-2012-0001 "National Excellence Program". This work was also supported by a grant of the Romanian National Authority for Scientific Research, CNCS-UEFISCDI (National Research Council-Executive Agency for Higher Education, Research, Development and Innovation Funding), project number PN-II-ID-PCE-2011-3-0442. In addition, the research grant nr. GTC_34027 is thanked, which was provided by the Babes-Bolyai 
University for young researchers. Furthermore, the authors would like to thank to the RomanianHungarian bilateral project nr. 661/2013/K-TÉT_12_RO-1-2013-0109966.

\section{Author Contributions}

The present paper is based on the research work of Zsejke Réka Tóth and Zsolt Pap. Characterization of the catalyst with photocatalytic measurements was carried out by Virginia Danciu. Planning of research strategy and writing of manuscript was carried out by Lucian Baia and Gábor Kovács.

\section{Conflicts of Interest}

The authors declare no conflict of interest.

\section{References}

1. Fujishima, A.; Honda, K.; Kikuchi, S. Photosensitized electrolytic oxidation on semiconducting n-type $\mathrm{TiO}_{2}$ electrode. J Soc. Chem. Ind. Jpn. 1969, 72, 108-113.

2. Fujishima, A.; Rao, T.N.; Tryk, D.A. Titanium dioxide photocatalysis. J. Photochem. Photobiol. C Photochem. Rev. 2000, 1, 1-21.

3. Gupta, S.M.; Tripathi, M. A review of $\mathrm{TiO}_{2}$ nanoparticles. Chin. Sci. Bull. 2011, 56, 1639-1657.

4. Rajbongshi, B.M.; Ramchiary, A.; Samdarshi, S.K. Influence of N-doping on photocatalytic activity of $\mathrm{ZnO}$ nanoparticles under visible light irradiation. Mater. Lett. 2014, 134, 111-114.

5. Siddiquey, I.; Furusawa, T.; Sato, M.; Suzuki, N. Microwave-assisted silica coating and photocatalytic activities of $\mathrm{ZnO}$ nanoparticles. Mater. Res. Bull. 2008, 43, 3416-3424.

6. Meng, Z.-D.; Zhu, L.; Oh, W.-C. Preparation and high visible-light-induced photocatalytic activity of CdSe and CdSe-C60 nanoparticles. J. Ind. Eng. Chem. 2012, 18, 2004-2009.

7. Ghosh, T.; Cho, K.-Y.; Ullah, K.; Nikam, V.; Park, C.-Y.; Meng, Z.-D.; Oh, W.-C. High photonic effect of organic dye degradation by $\mathrm{CdSe}-$ graphene- $\mathrm{TiO}_{2}$ particles. J. Ind. Eng. Chem. 2013, 19, 797-805.

8. Talebian, N.; Jafarinezhad, F. Morphology-controlled synthesis of $\mathrm{SnO}_{2}$ nanostructures using hydrothermal method and their photocatalytic applications. Ceram. Int. 2013, 39, 8311-8317.

9. Das, S.; Jayaraman, V. $\mathrm{SnO}_{2}$ : A comprehensive review on structures and gas sensors. Prog. Mater. Sci. 2014, 66, 112-255.

10. Farbod, M.; Meamar Ghaffari, N.; Kazeminezhad, I. Fabrication of single phase CuO nanowires and effect of electric field on their growth and investigation of their photocatalytic properties. Ceram. Int. 2014, 40, 517-521.

11. Zhang, Q.; Zhang, K.; Xu, D.; Yang, G.; Huang, H.; Nie, F.; Liu, C.; Yang, S. Cuo nanostructures: Synthesis, characterization, growth mechanisms, fundamental properties, and applications. Prog. Mater. Sci. 2014, 60, 208-337.

12. Kim, J.; Bondarchuk, O.; Kay, B.D.; White, J.M.; Dohnálek, Z. Preparation and characterization of monodispersed $\mathrm{WO}_{3}$ nanoclusters on $\mathrm{TiO}_{2}$ (110). Catal. Today 2007, 120, 186-195.

13. Song, X.C.; Zheng, Y.F.; Yang, E.; Wang, Y. Large-scale hydrothermal synthesis of $\mathrm{WO}_{3}$ nanowires in the presence of $\mathrm{K}_{2} \mathrm{SO}_{4}$. Mater. Lett. 2007, 61, 3904-3908. 
14. Ong, W.-J.; Tan, L.-L.; Chai, S.-P.; Yong, S.-T.; Mohamed, A.R. Facet-dependent photocatalytic properties of $\mathrm{TiO}_{2}$-based composites for energy conversion and environmental remediation. ChemSusChem 2014, 7, 690-719.

15. Vinu, R.; Madras, G. Renewable energy via photocatalysis. Curr. Org. Chem. 2013, 17, 2538-2558.

16. Daghrir, R.; Drogui, P.; Robert, D. Modified $\mathrm{TiO}_{2}$ for environmental photocatalytic applications: A review. Ind. Eng. Chem. Res. 2013, 52, 3581-3599.

17. Huang, C.-J.; Wang, Y.-H.; Chiu, P.-H.; Shih, M.-C.; Meen, T.-H. Electrochemical synthesis of gold nanocubes. Mater. Lett. 2006, 60, 1896-1900.

18. Baik, H.J.; Hong, S.; Park, S. Surface plasmon modes of gold nanospheres, nanorods, and nanoplates in an organic solvent: Phase-transfer from aqueous to organic media. J. Colloid Interface Sci. 2011, 358, 317-322.

19. Pérez-Juste, J.; Pastoriza-Santos, I.; Liz-Marzán, L.M.; Mulvaney, P. Gold nanorods: Synthesis, characterization and applications. Coord. Chem. Rev. 2005, 249, 1870-1901.

20. Mukherjee, P.; Roy, M.; Mandal, B.P.; Choudhury, S.; Tewari, R.; Tyagi, A.K.; Kale, S.P. Synthesis of uniform gold nanoparticles using non-pathogenic bio-control agent: Evolution of morphology from nano-spheres to triangular nanoprisms. J. Colloid Interface Sci. 2012, 367, 148-152.

21. Maity, D.; Gupta, R.; Gunupuru, R.; Srivastava, D.N.; Paul, P. Calix[4]arene functionalized gold nanoparticles: Application in colorimetric and electrochemical sensing of cobalt ion in organic and aqueous medium. Sens. Actuators Chem. 2014, 191, 757-764.

22. Benkovicova, M.; Vegso, K.; Siffalovic, P.; Jergel, M.; Luby, S.; Majkova, E. Preparation of gold nanoparticles for plasmonic applications. Thin Solid Films 2013, 543, 138-141.

23. Bindhu, M.R.; Umadevi, M. Antibacterial activities of green synthesized gold nanoparticles. Mater. Lett. 2014, 120, 122-125.

24. Orza, A.; Olenic, L.; Pruneanu, S.; Pogacean, F.; Biris, A.S. Morphological and electrical characteristics of amino acid-AuNP nanostructured two-dimensional ensembles. Chem. Phys. 2010, 373, 295-299.

25. Wang, J.-D.; Liu, J.-K.; Lu, Y.; Hong, D.-J.; Yang, X.-H. Catalytic performance of gold nanoparticles using different crystallinity hap as carrier materials. Mater. Res. Bull. 2014, 55, 190-197.

26. Omidfar, K.; Khorsand, F.; Darziani Azizi, M. New analytical applications of gold nanoparticles as label in antibody based sensors. Biosens. Bioelectron. 2013, 43, 336-347.

27. Bannat, I.; Wessels, K.; Oekermann, T.; Rathousky, J.; Bahnemann, D.; Wark, M. Improving the photocatalytic performance of mesoporous titania films by modification with gold nanostructures. Chem. Mater. 2009, 21, 1645-1653.

28. Primo, A.; Corma, A.; Garcia, H. Titania supported gold nanoparticles as photocatalyst. Phys. Chem. Chem. Phys. 2011, 13, 886-910.

29. Hidalgo, M.C.; Maicu, M.; Navío, J.A.; Colón, G. Effect of sulfate pretreatment on gold-modified $\mathrm{TiO}_{2}$ for photocatalytic applications. J. Phys. Chem. C 2009, 113, 12840-12847.

30. Veréb, G.; Ambrus, Z.; Pap, Z.; Kmetykó, Á.; Dombi, A.; Danciu, V.; Cheesman, A.; Mogyorósi, K. Comparative study on UV and visible light sensitive bare and doped titanium dioxide photocatalysts for the decomposition of environmental pollutants in water. Appl. Catal. A Gen. 2012, 417-418, 26-36. 
31. Iliev, V.; Tomova, D.; Bilyarska, L.; Tyuliev, G. Influence of the size of gold nanoparticles deposited on $\mathrm{TiO}_{2}$ upon the photocatalytic destruction of oxalic acid. J. Mol. Catal. A Chem. 2007, 263, 32-38.

32. Vijayakumar, S.; Paulsi, S. Gold nanoparticles in early detection and treatment of cancer: Biodistribution and toxicities. Int. J. Pharm. Sci. Rev. Res. 2013, 20, 80-88.

33. Kumar, D.; Saini, N.; Jain, N.; Sareen, R.; Pandit, V. Gold nanoparticles: An era in bionanotechnology. Expert Opin. Drug Deliv. 2013, 10, 397-409.

34. Katz, E.; Willner, I. Integrated nanoparticle-biomolecule hybrid systems: Synthesis, properties, and applications. Angew. Chem. Int. Ed. 2004, 43, 6042-6108.

35. Liu, X.Y.; Wang, A.; Zhang, T.; Mou, C.-Y. Catalysis by gold: New insights into the support effect. Nano Today 2013, 8, 403-416.

36. Ohtani, B.; Prieto-Mahaney, O.O.; Li, D.; Abe, R. What is Degussa (Evonik) P25? Crystalline composition analysis, reconstruction from isolated pure particles and photocatalytic activity test. $J$. Photochem. Photobiol. A Chem. 2010, 216, 179-182.

37. Petkov, V.; Peng, Y.; Williams, G.; Huang, B.; Tomalia, D.; Ren, Y. Structure of gold nanoparticles suspended in water studied by X-ray diffraction and computer simulations. Phys. Rev. B 2005, 72, doi:10.1103/PhysRevB.72.195402.

38. Kumar, D.V.R.; Kulkarni, A.A.; Prasad, B.L.V. Synthesis of triangular gold nanoplates: Role of bromide ion and temperature. Colloids Surf. A Physicochem. Eng. Asp. 2013, 422, 181-190.

39. Bernardi, M.; Raja, S.N.; Lim, S.K. Nanotwinned gold nanowires obtained by chemical synthesis. Nanotechnology 2010, 21, doi:10.1088/0957-4484/21/28/285607.

40. Karácsonyi, É.; Baia, L.; Dombi, A.; Danciu, V.; Mogyorósi, K.; Pop, L.C.; Kovács, G.; Coşoveanu, V.; Vulpoi, A.; Simon, S., et al. The photocatalytic activity of $\mathrm{TiO}_{2} / \mathrm{WO}_{3} /$ noble metal (Au or Pt) nanoarchitectures obtained by selective photodeposition. Catal. Today 2013, 208, 19-27.

41. Kuwauchi, Y.; Yoshida, H.; Akita, T.; Haruta, M.; Takeda, S. Intrinsic catalytic structure of gold nanoparticles supported on $\mathrm{TiO}_{2}$. Angew. Chem. Int. Ed. 2012, 51, 7729-7733.

42. Pap, Z.; Radu, A.; Hidi, I.J.; Melinte, G.; Diamandescu, L.; Popescu, T.; Baia, L.; Danciu, V.; Baia, M. Behavior of gold nanoparticles in a titania aerogel matrix: Photocatalytic activity assessment and structure investigations. Chin. J. Catal. 2013, 34, 734-740.

43. Maicu, M.; Hidalgo, M.C.; Colon, G.; Navio, J.A. Comparative study of the photodeposition of Pt, $\mathrm{Au}$ and $\mathrm{Pd}$ on pre-sulphated $\mathrm{TiO}_{2}$ for the photocatalytic decomposition of phenol. J. Photochem. Photobiol. A Chem. 2011, 217, 275-283.

44. Baia, L.; Vulpoi, A.; Radu, T.; Karácsonyi, É.; Dombi, A.; Hernádi, K.; Danciu, V.; Simon, S.; Norén, K.; Canton, S.E., et al. $\mathrm{TiO}_{2} / \mathrm{WO}_{3} / \mathrm{Au}$ nanoarchitectures' photocatalytic activity "from degradation intermediates to catalysts' structural peculiarities", part II: Aerogel based composites - fine details by spectroscopic means. Appl. Catal. B Environ. 2014, 148-149, 589-600.

45. Kovács, G.; Baia, L.; Vulpoi, A.; Radu, T.; Karácsonyi, É.; Dombi, A.; Hernádi, K.; Danciu, V.; Simon, S.; Pap, Z. $\mathrm{TiO}_{2} / \mathrm{WO}_{3} / \mathrm{Au}$ nanoarchitectures' photocatalytic activity, "from degradation intermediates to catalysts' structural peculiarities", part I: Aeroxide P25 based composites. Appl. Catal. B Environ. 2014, 147, 508-517. 
46. Ismail, A.A.; Bahnemann, D.W.; Bannat, I.; Wark, M. Gold nanoparticles on mesoporous interparticle networks of titanium dioxide nanocrystals for enhanced photonic efficiencies. J. Phys. Chem. C 2009, 113, 7429-7435.

47. Zhang, H.; Banfield, J.F. Understanding polymorphic phase transformation behavior during growth of nanocrystalline aggregates: Insights from $\mathrm{TiO}_{2}$. J. Phys. Chem. B 2000, 104, 3481-3487.

48. Jenkins, R.; Snyder, R.L. Introduction to X-Ray Powder Diffractometry. John Wiley \& Sons: New York, NY, USA, 1996.

(C) 2014 by the authors; licensee MDPI, Basel, Switzerland. This article is an open access article distributed under the terms and conditions of the Creative Commons Attribution license (http://creativecommons.org/licenses/by/4.0/). 